\section{Ultrasonographic measurement of the axillary recess thickness in an asymptomatic shoulder}

\author{
Gi-Young Park, Jin Hoon Lee, Dae Gil Kwon
}

Department of Rehabilitation Medicine, Catholic University of Daegu School of Medicine, Daegu, Korea

Purpose: The purpose of this study was to measure the axillary recess (AR) thickness in an asymptomatic shoulder by using ultrasonography (US) and to analyze the factors affecting it.

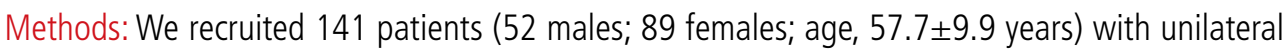
shoulder pain and performed US on the unaffected shoulder. Two physiatrists measured the AR thickness of the unaffected shoulder independently. All patients were examined in an upright sitting position with $90^{\circ}$ shoulder abduction. The ultrasonographic transducer was placed longitudinally on the mid-axillary line and along the long axis of the humeral shaft. The factors affecting the AR thickness values were analyzed, and intra-class correlation coefficients were used for assessing the reproducibility of each measurement.

Results: The intrarater reliability values for the two physiatrists were 0.98 and 0.96 , respectively. The inter-rater reliability of the mean $A R$ thickness measurements was 0.91 . The mean $A R$ thickness in all subjects, males, and females was $2.8 \pm 0.6 \mathrm{~mm}, 3.1 \pm 0.6 \mathrm{~mm}$, and $2.6 \pm 0.5 \mathrm{~mm}$ $(P<0.01)$, respectively. No difference between the left and the right sides (males, $P=0.086$; females, $\mathrm{P}=0.535$ ) or between the dominant and the non-dominant sides (males, $\mathrm{P}=0.173$; female, $P=0.244)$ was found. The AR thickness correlated positively with the height $(r=0.313$, $P<0.01)$ and the weight $(r=0.319, P<0.01)$. However, it did not correlate with the body mass index $(r=0.152, P=0.077)$ or the age $(r=-0.056, P=0.515)$.

Conclusion: US measurements of the AR thickness in asymptomatic shoulders demonstrated excellent intrarater and inter-rater reliabilities. The AR thickness showed anatomical variation with sex, height, and weight.

Keywords: Shoulder; Axilla; Ultrasonography

\section{Introduction}

Frozen shoulder (FS), also known as adhesive capsulitis, is a common and painful condition associated with the limitation of motion involving the scapulohumeral joint [1]. Neviaser initially used the term adhesive capsulitis on the basis of surgical findings that described capsular and synovial inflammation and adhesions leading to the adherence of the axillary fold and the anatomic neck of the humerus [2]. Inferior capsule thickening and contracture rather than the axillary fold adherence were observed

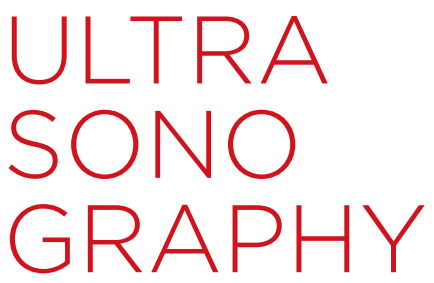

ORIGINAL ARTICLE

http://dx.doi.org/10.14366/usg. 16032 pISSN: 2288-5919 • elSSN: 2288-5943 Ultrasonography 2017;36:139-143

Received: July 29, 2016

Revised: September 15, 2016 Accepted: September 19, 2016

Correspondence to: Dae Gil Kwon, MD, Department of Rehabilitation Medicine, Catholic University of Daegu School of Medicine, 33 Duryugongwon-ro 17-gil, Nam-gu, Daegu 42472, Korea

Tel. +82-53-650-4894

Fax. +82-53-622-4687

E-mail: cateyesn@naver.com

This is an Open Access article distributed under the terms of the Creative Commons Attribution NonCommercial License (http://creativecommons.org/ licenses/by-nc/3.0//) which permits unrestricted noncommercial use, distribution, and reproduction in any medium, provided the original work is properly cited.

Copyright (C) 2017 Korean Society of Ultrasound in Medicine (KSUM)

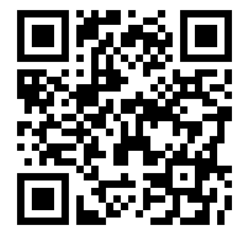

How to cite this article:

Park GY, Lee JH, Kwon DG. Ultrasonographic measurement of the axillary recess thickness in an asymptomatic shoulder. Ultrasonography. 2017 Apr;36(2):139-143 
in patients with FS [3]. On the other hand, FS was associated with significant differences in rotator interval dimensions and axillary pouch thickness values [4]. The diagnosis of FS might be difficult because many painful shoulder conditions have similar clinical symptoms [5]. Therefore, imaging studies including arthrography, ultrasonography (US), and magnetic resonance imaging (MRI) have been used. Currently, shoulder arthrography is considered the gold standard for the imaging diagnosis of FS. Arthrography in FS revealed a decreased capacity of less than $10 \mathrm{~mL}$ at the glenohumeral joint cavity, with the obliteration of the axillary and subscapular recesses and the long biceps tendon sheath [3]. However, this is an invasive diagnostic method involving radiation exposure and cannot explore pathologic changes in the synovial and capsular membranes.

Even though FS pathophysiology remains unclear, abnormalities in the synovial layer and the joint capsule have been reported in several histologic studies [6]. MRI suggests that FS involves inflammatory and/or degenerative changes of the glenohumeral joint capsule associated with increased vascularity in the synovial and/or subsynovial layer, which leads to the thickening and shrinking of the capsule and the formation of adhesions [6].

We anticipate that the US measurement of the axillary recess (AR) thickness would be a useful imaging method in the diagnosis of FS. Prior to evaluating AR thickness in patients with FS, the AR thickness must be measured in asymptomatic subjects. However, the US measurement of the AR thickness in asymptomatic shoulders has not been reported in the literature. The purpose of this study is to measure the AR thickness by using US in asymptomatic shoulders, to assess the inter-rater and intrarater reliabilities of the US measurement of AR thickness, and to analyze the subject characteristics affecting the AR thickness.

\section{Materials and Methods}

\section{Patients}

One hundred and forty-one patients (52 males, 89 females; mean age, $57.7 \pm 9.9$ years; range, 27 to 83 years) with unilateral shoulder pain (54 right, 87 left) were recruited at the outpatient department of the university hospital. One hundred and thirty-three patients were right-side dominant, three patients were left-side dominant, and five patients were mixed dominant. Fifty-six shoulders were the dominant side, and 80 shoulders were the non-dominant side. The Institutional Review Board and Ethics Committee approved our study protocol.

Patients with previous shoulder surgery or trauma, partial or full-thickness rotator cuff tendon tear or calcific tendinitis on US, inflammatory arthritis, osteoarthritis, neurological disorders of the upper limbs, and abnormal shoulder radiographs in unaffected shoulders were not enrolled in the study. At the beginning, 32 (13 males, 19 females; mean age, $53.7 \pm 9.9$ years; range, 27 to 70 years) of the 141 patients were recruited to evaluate the inter-rater and intrarater reliabilities of the US measurement because a prior power analysis for the intra-class correlation coefficient (ICC) based on the pilot results determined that 32 subjects would yield a power of 0.9 at a significance level of 0.01 .

\section{US Measurement}

AR thickness was measured using US in both shoulders by a physiatrist with 20 years of musculoskeletal US experience using a commercially available US system with an 18-5-MHz multifrequency linear transducer (EPIQ 5, Philips Healthcare, Andover, MA, USA). The AR thickness of the unaffected shoulder was measured twice independently by two physiatrists with 20- and 1-year musculoskeletal US experience for the inter-rater and intrarater reliabilities of US measurement. All patients were examined in the upright sitting position with $90^{\circ}$ shoulder abduction on the basis of Koski's study (Fig. 1A) [7]. The transducer was placed longitudinally on the mid-axillary line and along the long axis of the humeral shaft. After the humeral head and the surgical neck were identified on the longitudinal scan, the AR thickness was measured perpendicularly in the thickest AR portion in the middle of the humeral surgical neck concavity (Fig. 1B). The mean AR thickness measured in 141 unaffected shoulders by the physiatrist was used for the analysis of the factors affecting the AR thickness.

\section{Statistical Analysis}

Statistical analysis was performed using SPSS ver. 19.0 (IBM Co., Armonk, NY, USA) with the significance level set at $<0.05$. The ICC was used for examining the intrarater and inter-rater reliabilities of the repeated AR measurements between two physiatrists. An independent $t$ test was used for assessing the AR thickness difference between males and females. The independent $t$ test was also used for assessing the AR thickness difference between the right and the left sides, and between the dominant and the nondominant sides for each sex. The correlation between the AR thickness and age and the body mass index (BMI), weight, and height was determined using Pearson's correlation coefficient.

\section{Results}

The mean AR thickness of 141 unaffected shoulders was $2.8 \mathrm{~mm}$ (range, 1.4 to $5.2 \mathrm{~mm}$ ). The ICC of the repeated AR thickness measurements by the two physiatrists was 0.98 and 0.96 , respectively. The ICC of the mean AR thickness measurement 


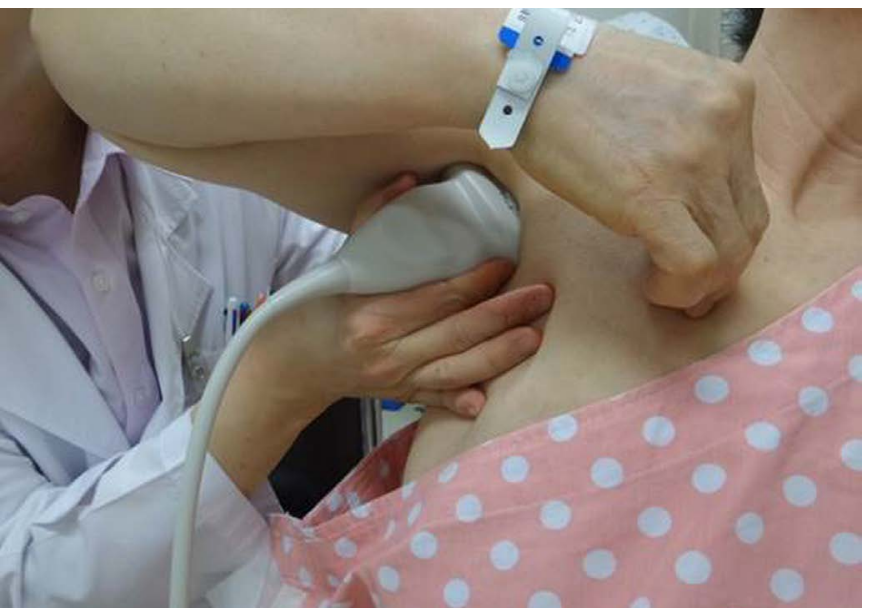

A

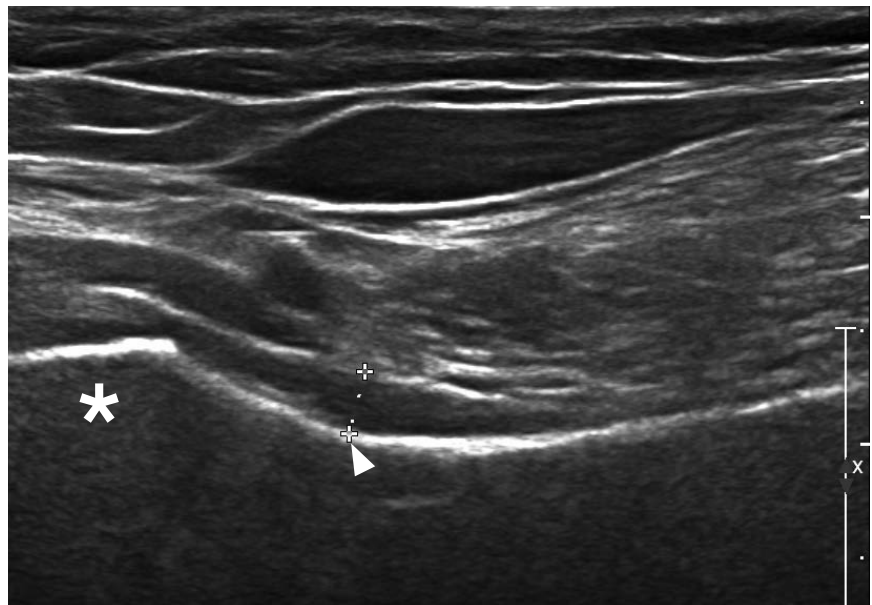

B

Fig. 1. Measurement of the axillary recess thickness.

A. Ultrasonographic probe is positioned in the axilla. B. A representative longitudinal sonogram shows the measurement of the axillary recess thickness. The thickness (crosshairs) was measured perpendicularly in the thickest portion of axillary recess at the humeral surgical neck (arrowhead). Asterisk, humeral head.

between the two physiatrists was 0.91 .

The mean AR thickness $(3.1 \pm 0.6 \mathrm{~mm})$ in males was significantly greater than that in females $(2.6 \pm 0.5 \mathrm{~mm})(P<0.01)$ (Table 1). No difference was found in the AR thickness between the left and the right shoulders in the male (left $3.0 \pm 0.5$ vs. right $3.3 \pm 0.8 \mathrm{~mm}$, $P=0.086$ ) and female groups (left $2.6 \pm 0.5$ vs. right $2.5 \pm 0.5 \mathrm{~mm}$, $P=0.535)$. There was no difference in the AR thickness between the dominant side and the non-dominant side in the male (dominant side $3.3 \pm 0.8$ vs. non-dominant side $3.0 \pm 0.5 \mathrm{~mm}, \mathrm{P}=0.173)$ and the female groups (dominant side $2.6 \pm 0.5$ vs. non-dominant side $2.5 \pm 0.5 \mathrm{~mm}, \mathrm{P}=0.244)$. The mean AR thickness correlated positively with the height $(r=0.313)$ and the weight $(r=0.319)$. However, the mean AR thickness did not correlate with BMI $(r=0.152)$ and age $(r=-0.056)$ (Table 2).

\section{Discussion}

We measured the mean AR thickness on US and found excellent intrarater and inter-rater reliabilities of the US measurement of AR thickness; we also found significant differences in the AR thickness by comparing US measurements among subjects of different sex, height, and weight. This is the first report to present such findings.

Some studies measured the AR thickness by using MRI of normal shoulders, and the mean thickness of the capsule and synovium at the AR ranged from 1 to $4.2 \mathrm{~mm}[5,8-10]$. The main reason for the wide variation in the normal value of the AR thickness on MRI is the different methods of measurement. In some studies, the widest portion of the low-signal region equivalent to the capsule and the
Table 1. Ultrasonographic measurement of the AR thickness in a normal shoulder

\begin{tabular}{cccc}
\hline & Male $(\mathrm{n}=52)$ & Female $(\mathrm{n}=89)$ & Total $(\mathrm{n}=141)$ \\
\hline AR thickness $\left.(\mathrm{mm})^{\mathrm{a}}\right)$ & $3.1 \pm 0.6$ & $2.6 \pm 0.5$ & $2.8 \pm 0.6$
\end{tabular}

AR, axillary recess.

${ }^{a)} P<0.01$, significant difference between male and female by independent $t$ test.

Table 2. Correlation between the AR thickness and the patient characteristics

\begin{tabular}{lcccc}
\hline & \multicolumn{4}{c}{ Patient characteristic } \\
\cline { 2 - 5 } & Age & BMI & Height & Weight \\
\hline AR thickness & -0.056 & 0.152 & $0.313^{\text {a) }}$ & $0.319^{\text {a) }}$ \\
\hline AR, axilary
\end{tabular}

$A R$, axillary recess; $B M I$, body mass index.

${ }^{a)} P<0.01$, Pearson's correlation coefficient.

synovium between the high-signal fluid in the AR and the low-signal cortical bone of the humerus was measured $[5,8]$. In other studies, the thickest area or enhancing portion of the AR on the oblique coronal images was measured at the widest portion of the humeral and glenoid aspects of the AR $[9,10]$.

However, a considerable difference among the studies was observed although the same method was used for measuring the AR thickness. Manton et al. [11] measured the widest portion of the joint capsule/synovium at the AR on coronal fat-suppressed T1weighted images at the medial aspect of the humeral head and its insertion at the humeral head in a normal shoulder and reported that the mean AR thickness was $0.4 \mathrm{~mm}$ (range, 0.0 to $2.0 \mathrm{~mm}$ ). 
Emig et al. [8] reported that the mean AR thickness was $2.9 \mathrm{~mm}$ (range, 2.0 to $3.8 \mathrm{~mm}$ ) in a normal shoulder by using the method of Manton et al. [11]. There are two explanations for this difference. First, MRI has a potential limitation on measurement accuracy. The pixel size in the MRI was approximately $0.5 \mathrm{~mm}$, which was larger in the measurement of anatomic structures by several millimeters [8]. Second, subject characteristics such as age, sex, height, and weight that may influence the AR thickness were not considered.

The mean AR thickness on the US measurement in asymptomatic shoulders was $2.8 \mathrm{~mm}$ (range, 1.4 to $5.2 \mathrm{~mm}$ ) in our study. Our result is similar to that of Emig's study that reported a value of 2.9 $\mathrm{mm}$ (range, 2.0 to $3.8 \mathrm{~mm}$ ) through an MRI measurement [8].

The AR thickness on the US measurement in our study was associated with a subject's characteristics including sex, height, and weight. The mean AR thickness was significantly greater in men than in women. In previous MRI studies, the difference in the AR thickness between the sexes was not assessed, and they reported that the capsule and synovium thickness at the AR greater than $4 \mathrm{~mm}$ was a specific (95\%) and sensitive (70\%) criterion for the diagnosis of adhesive capsulitis [8]. Based on our result, the AR thickness difference between the sexes should be considered on MRI that only measures the AR thickness in the affected shoulder. The US measurement of the AR thickness correlated positively with the height and weight in all subjects. However, the BMI did not correlate with the AR thickness. These findings indicated that the AR thickness could be influenced by the height and weight of a subject, but not obesity. Because the AR thickness varied with sex, weight, and height, the measurement of the absolute AR thickness is not recommended as a good diagnostic indicator for FS. Instead, the ratio of $A R$ thickness between the affected and the unaffected sides could be a reliable diagnostic parameter in patients with unilateral FS. US measurement of the AR thickness revealed excellent intrarater and inter-rater reliabilities irrespective of the musculoskeletal US experience in our study. Our results are superior to the reliability in MRI, which is approximately 0.8 [6]. Therefore, the measurement of the AR thickness can be used as a reliable US method.

Previous studies proposed the usefulness of $A R$ thickness measurement using MRI for the diagnosis of FS $[4,5,8-11]$. Lee et al. [5] reported that the thickness of the joint capsule and synovium on MRI was significantly different between patients with and without adhesive capsulitis. In contrast, Manton et al. [11] reported that capsular and synovial thickness and joint volume were inconclusive as MR arthrographic signs for distinguishing shoulders with adhesive capsulitis from those without.

US possesses several obvious advantages over MRI for the measurement of AR thickness in patients with FS. First, US examinations are inexpensive. Therefore, this approach can be cost- effective and widely available. Second, US imaging easily provides a comparison between the affected and the unaffected sides. However, MRI cannot be performed bilaterally. Third, US can measure AR thickness up to $0.1 \mathrm{~mm}$. Lastly, MRI needs an intravenous contrast agent to visualize the axillary fold clearly.

The AR is folded and redundant in the neutral position [12]. The AR is stretched with a lateral elevation of an arm [12]. The AR thickness in the studies using MRI was measured at the widest portion of the capsule and synovium at its insertion at the humeral head perpendicular to the adjacent cortical bone $[6,8]$. This did not indicate the accurate AR thickness because this portion consists of two layers of the axillary fold (glenoidal and humeral aspect) and the joint fluid space. Other MRI studies measured each portion of the humeral and glenoid aspects of the AR $[13,14]$. This method did not obtain measurements at the midportion of the AR between the humeral and the glenoid aspects. On MRI, the measurement of the $A R$ at the midportion can be overestimated because AR is folded and redundant in the neutral position of the shoulder. Therefore, the best position to measure the entire AR thickness at the midportion of the axilla is the lateral elevation of the arm that can open and stretch the folding portion of AR.

In our study, US measurement was performed in patients in the upright sitting position with $90^{\circ}$ shoulder abduction, and the transducer was placed on the mid-axillary line and along the long axis of the humeral shaft.

Our study has a few limitations. First, we did not measure the AR thickness in normal subjects without shoulder pain. The unaffected shoulders in our study were strictly asymptomatic. Our subjects may have a degenerative change of shoulder structures because the mean age of the subjects was 57.7 years. Therefore, the AR thickness of an asymptomatic shoulder in our study needs to be cautiously considered to be the normal reference value before it can be used as such. Second, we did not measure the AR thickness in the shoulder position with different abduction angles. In our study, the AR thickness was measured at $90^{\circ}$ shoulder abduction because the transducer could be placed longitudinally at the midaxillary line in that position. Third, we did not consider the effect of systemic diseases such as diabetes mellitus and thyroid disease. The association between diabetes mellitus and thyroid disease, and adhesive capsulitis is well known $[15,16]$. However, there has been no research on the influence of systemic disease on the AR thickness of patients with $\mathrm{FS}$.

In conclusion, our results demonstrated excellent intrarater and inter-rater reliabilities for the US measurement of AR thickness in a normal shoulder. US imaging is a useful method that can measure AR thickness. In addition, AR thickness varies with sex, height, and weight. Therefore, understanding these thickness differences may 
improve the reliability of AR thickness as a diagnostic method.

ORCID: Gi-Young Park: http://orcid.org/0000-0003-0056-1556; Jin Hoon Lee: http://orcid.org/0000-0001-9725-9921; Dae Gil Kwon: http://orcid.org/0000-00028423-8045

\section{Conflict of Interest}

No potential conflict of interest relevant to this article was reported.

\section{References}

1. Hsu JE, Anakwenze OA, Warrender WJ, Abboud JA. Current review of adhesive capsulitis. J Shoulder Elbow Surg 2011;20:502-514.

2. Neviaser JS. Adhesive capsulitis of the shoulder: a study of the pathological findings in periarthritis of the shoulder. J Bone Joint Surg Am 1945;27:211-222.

3. Wiley AM. Arthroscopic appearance of frozen shoulder. Arthroscopy 1991;7:138-143.

4. Connell D, Padmanabhan R, Buchbinder R. Adhesive capsulitis: role of MR imaging in differential diagnosis. Eur Radiol 2002;12:21002106.

5. Lee MH, Ahn JM, Muhle C, Kim SH, Park JS, Kim SH, et al. Adhesive capsulitis of the shoulder: diagnosis using magnetic resonance arthrography, with arthroscopic findings as the standard. J Comput Assist Tomogr 2003;27:901-906.

6. Lefevre-Colau MM, Drape JL, Fayad F, Rannou F, Diche T, Minvielle $F$, et al. Magnetic resonance imaging of shoulders with idiopathic adhesive capsulitis: reliability of measures. Eur Radiol 2005;15:2415-2422.
7. Koski JM. Validity of axillary ultrasound scanning in detecting effusion of the glenohumeral joint. Scand J Rheumatol 1991;20:4951.

8. Emig EW, Schweitzer ME, Karasick D, Lubowitz J. Adhesive capsulitis of the shoulder: MR diagnosis. AJR Am J Roentgenol 1995; 164:1457-1459.

9. Gokalp G, Algin O, Yildirim N, Yazici Z. Adhesive capsulitis: contrastenhanced shoulder MRI findings. J Med Imaging Radiat Oncol 2011;55:119-125.

10. Song KD, Kwon JW, Yoon YC, Choi SH. Indirect MR arthrographic findings of adhesive capsulitis. AJR Am J Roentgenol 2011;197: W1105-W1109.

11. Manton GL, Schweitzer ME, Weishaupt D, Karasick D. Utility of MR arthrography in the diagnosis of adhesive capsulitis. Skeletal Radiol 2001;30:326-330.

12. Ryu KN, Lee SW, Rhee YG, Lim JH. Adhesive capsulitis of the shoulder joint: usefulness of dynamic sonography. J Ultrasound Med 1993;12:445-449.

13. Mengiardi B, Pfirrmann CW, Gerber C, Hodler J, Zanetti M. Frozen shoulder: MR arthrographic findings. Radiology 2004;233:486-492.

14. Jung JY, Jee WH, Chun HJ, Kim YS, Chung YG, Kim JM. Adhesive capsulitis of the shoulder: evaluation with MR arthrography. Eur Radiol 2006;16:791-796.

15. Cakir M, Samanci N, Balci N, Balci MK. Musculoskeletal manifestations in patients with thyroid disease. Clin Endocrinol (Oxf) 2003;59:162-167.

16. Pal B, Anderson J, Dick WC, Griffiths ID. Limitation of joint mobility and shoulder capsulitis in insulin- and non-insulin-dependent diabetes mellitus. Br J Rheumatol 1986;25:147-151. 